\title{
Posterolateral decompression, distractive reduction and reconstructive stabilization for the treatment of thoracic and lumbar spine fracture-dislocations
}

Changbao Chen

Tianjin Hospital

Xiaolin Zhang

Tianjin Hospital

Xinlong Ma ( $\nabla$ richivaldchen@gmail.com )

Tianjin Hospital

Research article

Keywords: Posterolateral decompression, Distractive reduction, Reconstructive stabilization, Thoracic and lumbar spine fracture-dislocations.

Posted Date: March 24th, 2020

DOI: https://doi.org/10.21203/rs.3.rs-18702/v1

License: (c) (i) This work is licensed under a Creative Commons Attribution 4.0 International License. Read Full License 


\section{Abstract}

Background: Fracture-dislocations of the thoracic and lumbar spine are uncommon and highly unstable injuries, and the ideal management for patients with thoracic and lumbar spine fracture-dislocations is challenging. This study was designed to evaluate the clinical and radiographic results following posterolateral decompression, distractive reduction and reconstructive stabilization for patients with severe thoracic and lumbar spine fracture-dislocations.

Methods: Twenty-three patients with thoracic and lumbar spine fracture-dislocations underwent this procedure. Demographic data, radiographic results, neurologic function, clinical functions and treatmentrelated complications were prospectively evaluated.

Results: The average preoperative sagittal and coronal displacement was $31.9 \%$ and $6.7 \%$, significantly improved to $6.4 \%$ and $2.1 \%$ after surgery, respectively. The final sagittal and coronal displacement was $5.3 \%$ and $1.5 \%$. The average segmental kyphosis was $20.4^{\circ}$ before surgery, markedly corrected to $4.7^{\circ}$ immediately after surgery. The final sagittal kyphosis was $6.1^{\circ}$. Twelve patients with incomplete neurologic deficits had improvement by at least one ASIA grade neurologic improvement. The preoperative pain level showed a mean VAS score of 9.5 improved to 3.5 postoperatively, and to 1.2 at the final follow-up. Mean ODI preoperatively was $90.2 \%$, and improved to $38.3 \%$ postoperatively, and to $24.9 \%$ at the final follow-up. No patient had persistent postoperative back pain. No obvious complications were observed in this series.

Conclusions: Posterolateral decompression, distractive reduction and reconstructive stabilization is a reasonably safe and effective technique for thoracic and lumbar spine fracture-dislocations. This replicable procedure can result in satisfactory clinical and radiographic outcomes, with sufficiently circumferential decompression, excellent reduction, perfect spine alignment, solid instrumentation and sound fusion.

\section{Introduction}

Fracture-dislocations of the thoracic and lumbar spine are highly unstable injuries, frequently resulting in neurologic injuries in 75\% or more of patients [1]. The injury morphology of fracture-dislocations is commonly identified as translational injury in Thoracolumbar Injury Classification and Severity Score (TLICS) [2], or type C injury in AOSpine Thoracolumnbar Spine Injury Classification System [3]. These injuries are accompanied by combination of fracture, displacement/translation, disc disruption and ligamentous complex injury [4]. The ideal surgical strategy and surgical approaches such as anterior, posterior, and combined approaches for fracture-dislocations remains controversial, although the surgical techniques and instruments have been well identified [5]. Recently, a posterior-only approach may allow spine surgeons to access the ventral thoracic and lumbar spine, which has gained recognition for circumferentially decompressing the neural elements and reconstructing the anterior and middle columns 
in patients with spine trauma, deformity and tumors [6-8], having produced satisfactory clinical and radiographic outcomes in patients with unstable thoracolumbar fracture $[8,9]$.

In this report, we introduce a single-stage posterolateral decompression, distractive reduction and reconstructive stabilization technique via a posterior-only approach for 23 cases with thoracic and lumbar spine fracture-dislocations in the acute phase to achieve decompression of the neural elements, reduction of the displacement/translation, restoration of sagittal and coronal alignment, reconstructive stabilization with sound fusion.

\section{Materials And Methods}

\section{Study population}

Patients with the thoracic and lumbar fracture-dislocations were prospectively collected at the department of spinal surgery in our hospital. Our indications for surgery were spinal instability and/or neurologic injury. The average follow-up was 54 months (range 6-84 months). The inclusion criteria were as follows: (1) a fracture-dislocation of the thoracic or lumbar spine (AOSpine Type C) with or without neurologic injury [4]; (2) a fracture severity score point 8 or more graded using the thoracolumbar injury classification and severity score (TLICS) [3]. The exclusion criteria were: (1) a stable fracture of the thoracic or lumbar spine including compression and burst fracture; (2) an unstable burst fracture (AOSpine Type A) or combined with posterior ligamentous complex injuries (AOSpine Type B); (3) skeletal immaturity, a senile, osteopenic or insufficiency fracture; and (4) multi-segmental spinal injuries. These criteria were suitable to 23 patients, and the medical records, radiographic images including X-rays, threedimensional CT reconstruction scan, CT angiography and MRI, and neurologic status determined by the American Spinal Injury Association (ASIA) Impairment Scale [10], were accessible for all. All patients were included based on written informed consent. Institutional review board approval and patient informed consent to participate in this study were obtained.

\section{Surgical Technique}

All operations were successfully performed by the same surgeon using the same surgical procedures as presented in Fig. 1.

Incision, exposure and long instrumentation. All patients were placed prone. Through a standard posterior midline approach, the vertebral posterior arches to be instrumented were fully exposed. The transpedicular Schanz screws (AO USS; Synthes GmbH, Bettlach, Switzerland) with double thread for reduction were inserted into the cranial vertebra cephalad to the dislocated vertebra, whereas normal Schanz screws were inserted into the caudal vertebra caudad to the dislocated vertebra.

Posterolateral decompression and release. The process spinous and any accessible bone fragments were carefully removed using rongeur. Then a high-speed drill with burr attached was used to thin the lamina. Both lamina of the fractured vertebra and the cranial vertebra were completely removed using 
1.5- to $3 \mathrm{~mm}$ Kerrison rongeurs to decompress the posterior aspect of the thecal sac. The facet and pars interarticularis were carefully removed to facilitate reduction. For reverse cortical sign of the posterior wall fragment, we performed the direct posterolateral circumferental decompression: a laminectomy had been performed followed by unilateral excision of the facet and pedicle. A high-speed burr and/or angled curette were used to widen the pedicle channel and enter the fractured vertebral body, forming a recess beneath the retropulsed fragment. The retropulsed fragment was tamped down into the recess and take out, clearing the spinal canal.

Distractive reduction and reconstruction of anterior column. We assembled the short temporary rod and distraction: place the fracture clamps on the Schanz screws at 1 level above and below the dislocated vertebra, and secure caudal fracture clamps to the temporary rod and maintain the cranial fracture clamps not to the temporary rod. Fix the fracture clamps on the vertical rods, and tighten the caudal set screws to the temporary rod. Distraction with the spreader forceps under direct vision was carefully performed to identify the gap between the cranial and caudal dislocated vertebra. After sufficient posterolateral decompression and release, the enhanced gap between the cranial and caudal dislocated vertebra was significantly evident to facilitate the reduction of the dislocated vertebra. Secure the caudal and cranial fracture clamps to the temporary rod, and slide the USS reduction sleeves and knurled nuts over the Schanz screws with double thread. Distraction with the spreader forceps was accomplished under direct vision. Turn the nuts on both sides until the desired reduction was achieved under fluoroscopy guidance. And then, place the cannulated socket wrenches bilaterally over the cranial and caudal Schanz screws and clamps. Tilt both wrenches cranially and caudally to reduce the anterior column and lordose the spine, respectively. Secure the desired angulation by tightening the nut on the clamp with the socket wrench. Remove the USS knurled nuts and tighten the fracture clamps using the socket wrench. The final titanium final rods with appropriate length were precontoured to the appropriate anatomic sagittal profile and were installed on both sides. Fix fracture clamps on the final rods and trim Schanz screws. Cross-links were not routinely used. We did routinely perform long posterolateral fusion for these patients with translational injury. If the fractured vertebral body is significantly comminuted and fragment displacement is conspicuous, the anterior column should be reconstructed with posterior corpectomy and implanted an autograft-filled titanium mesh. Discectomies are then performed cephalad and caudad to the fractured vertebra. The ipsilateral pedicle is completely resected. Through the transpedicular space, resection of the posterior two thirds of the fractured body can be accomplished with curettes, rongeurs, or a high-speed burr, which serves to decompress the spinal canal as well as prepare the space for mesh placement. The mesh should be positioned in direct contact with end plates of the adjacent intact vertebra. Compression across the posterior pedicle screws establishes final correction of sagittal alignment.

Wound Closure. The wound was closed in layers and a closed suction drain was left in place for 48-72 hours according to drainage volume. For cerebrospinal fluid leakage, suction drain was kept for at least 3 days until drainage volume was less than $50 \mathrm{ml}$. 
Postoperative care and follow-up evaluation. All patients were encouraged to sit after the first week postoperatively. The vertebral body sagittal and coronal translation percentage and sagittal kyphosis by measuring the Cobb angle were determined as recommended by the Spine Traum Study Group [11]. Back pain and functional evaluations were determined on the basis of Visual Analog Scale (VAS) and Oswestry Disability Index score (ODI), respectively [12].

\section{Statistical analysis}

Continuous variables were presented as mean \pm standard deviation, and differences were analyzed by Student $t$ test. Statistical analyses were performed by SPSS software (SPSS, Chicago, IL). The level of significance was set at $P<0.05$. All reported $P$ values were two-sided.

\section{Results}

\section{Clinical characteristics of the study population}

The detailed clinical characteristics of all patients were shown in Table 1. The male-to-female ratio was $21: 2$ and the mean age was 39.7 years. Fall from height was the most common aetiological factor. The most injury level was T11/12 segment. The average points of thoracolumbar injury classification and severity score (TLICS) were 8.6 points. Eight patients were recognized as paraplegic (ASIA A) and the prevalence rate was $34.8 \%$. In the setting of significantly comminuted burst fractures with conspicuous fragment displacement, three patients (case 19, 22, 23) underwent posterior corpectomy and implanted an autograft-filled titanium mesh to achieve anterior column stabilization. In this series, the average injury-surgery interval was 11 days, and the mean operation time was $243.7 \mathrm{~min}$. The average estimated blood loss was $982.1 \mathrm{ml}$, and the mean hospital stay was 32 days. The average follow-up was 54 months.

\section{Assessment of radiographic outcomes}

Two-plane displacements were found in ten cases in this series. The average preoperative sagittal and coronal displacement was $31.9 \%$ and $6.7 \%$, which significantly improved to $6.4 \%$ and $2.1 \%$ after surgery $(P<0.001)$, respectively. The final sagittal and coronal displacement was $5.3 \%$ and $1.5 \%$. Correction loss of sagittal and coronal displacement was $1.1 \%$ and $0.6 \%$, and no substantial residual displacement was detected. The average sagittal kyphotic angle was $20.4^{\circ}$ before surgery, which was markedly corrected to $4.7^{\circ}$ immediately after surgery $(P<0.001)$. The final sagittal kyphosis was $6.1^{\circ}$. Loss of kyphosis correction was $1.4^{\circ}$, and no significant residual kyphosis was detected. Tables 2 and 3 show the detailed radiographic data.

\section{Neurologic recovery and clinical functions}

Neurologic injuries were detected in all patients. Twelve patients with incomplete neurologic deficits had improvement by at least one ASIA grade neurologic improvement on the final follow-up observation, with 2 (18.2\%) patients demonstrating complete neurologic recovery (ASIA E). Two patients with 
complete paralysis (ASIA A) showed no improvement in neurological function during follow-up, whereas nine patients experienced minor improvement of less than one ASIA grade, presenting as relieved numbness and partial muscle strength improvement. No neurological deterioration was observed in this series (Table 4).

All patients exhibited excellent improvement of pain and function after surgery (Table 5 ). The preoperative pain level showed a mean VAS score of 9.5 improved to 3.5 postoperatively, and to 1.2 at the final follow-up $(P<0.01)$. Mean ODI preoperatively was $90.2 \%$, and improved to $38.3 \%$ postoperatively, and to $24.9 \%$ at the final follow-up $(P<0.01)$. No patient had persistent postoperative back pain.

\section{Complications}

All patients had uneventful postoperative recoveries with no obvious complications in this series. Three patients (case $4,11,22$ ) suffered from cerebrospinal fluid (CSF) leakage due to dural lacerations caused by laminar fracture, who were cured with conservative treatment. No postoperative infection related to surgery developed in all cases. No cases of implant failure, including breakage, bending, or loosening of the pedicle screw, were observed at the follow-up period.

\section{Discussion}

The vertebral displacement/translation and disruption of the posterior ligamentous complex make the thoracic and lumbar fracture-dislocations the most unstable spinal injuries, obviously requiring surgical intervention to decompress the neural elements and reconstruct stabilization of the spine [2-4]. To date, no conclusive clinical studies are available to help spine surgeons decide on the optimal surgical management for thoracic and lumbar fracture-dislocations $[13,14]$. In terms of surgical approach, thoracic and lumbar fracture-dislocations can be surgically managed via posterior or combined approaches, because severe spine malalignment in two planes is difficult to restore and maintain through a single anterior approach $[5,15]$. Traditionally, combined approaches are thought to afford better decompression, superior biomechanical stability with anterior column reconstruction, and a limited number of mobile segments as compared to the posterior-only approach $[16,17]$. However, this combined approach has a higher risk of intra- and post-operative complications including more trauma, longer operation times, more blood loss and greater costs than the posterior approach alone [16]. Notably, the posterolateral approach has gained recognition for circumferentially decompressing the neural elements and reconstructing the anterior columns [18]. Recently, Yadla et al [19] used the combination approach to treat five cases of serious fractures-dislocations at the thoracolumbar junction, and found three cases with complications. In a retrospective study, Lu et al [20] found that the morbidity of the posterior approach was lower than that of an anterior-posterior combined approach. In our cohort, all patients were operated with a posterior-only approach with the avoidance of an anterior approach, through which dislocated vertebra can be effectively reduced and fractured vertebral body height can be significantly achieved using distraction and reduction by ligamentotaxis as shown in Fig. 2. However, our series is small and more in-depth analysis of a larger patient population is needed. We believe that, for certain 
carefully selected cases of thoracic and lumbar fracture-dislocations, a single-stage posterior-only approach may be preferred over the combined posterior-anterior approach, accompanied with fewer complications.

Anterior column reconstruction via a posterior-only approach maintains most of the advantages related to the anterior or combined approach while obviating the inherent risks $[6,8,9,21]$. Schmid $\mathrm{R}$ et al [21] reported satisfactory anterior column reconstruction with monocortical strut grafts via a PLIF (Posterior Lumbar Interbody Fusion) technique in 100 patients with thoracolumbar fractures. Yang et al [8] and Sasani et al [9] introduced posterior corpectomy and the three-column reconstruction technique via an exclusively posterior approach in unstable thoracolumbar fractures. Notably, supplementation of the anterior column by posterior corpectomy and implanting graft, cage or mesh is debatable [14]. In this series, in 3 cases with significant impairment of the anterior column that presented with a comminuted split fracture and conspicuous fragment displacement, we chose a partial corpectomy to circumferentially decompress the neural elements and reconstructed anterior column by implanting an autograft-filled titanium mesh. No obvious complications were observed in these cases. In our experience, after temporary fixation and distractive reduction, we can restore the fractured vertebral body height and reconstruct the anterior column stability. If the fractured vertebral body morphology was not satisfactorily achieved using a distractive reduction and ligamentotaxis technique, we can perform subtotal corpecotmy and rapidly prepare for anterior reconstruction by implanting an autograft-filled titanium mesh. However, we did routinely not performed posterior corpectomy and anterior column reconstruction because these procedures may remarkably enlong operation time, increase blood loss and surgical infection rate. Therefore, posterior corpectomy with circumferential reconstruction was reserved for patients with significantly comminuted split fracture and conspicuous fragment displacement.

For these severe thoracic and lumbar fracture-dislocations, we routinely chose long-segment posterior transpedicular constructs that fixed the affected level at two levels above and below it, because it can serve well in fracture-dislocations of thoracic and lumbar spine biomechanically [22, 23]. Short temporary rod was used to facilitate distractive reduction as shown in Fig. 1. Long-segment constructs afford a long lever arm for reduction, especially important in coronal plane realignment. Previous reports suggest that treatment with short-segment pedicle fixation has a reported failure rate of $26 \%$ in the thoracolumbar region, and an even higher rate of $83 \%$ in the lower lumbar spine [23]. Moreover, long segment fixation is necessary to disperse the stress and reduce the hardware failure rate. In this report, we obtained stable biomechanical reconstruction in all patients with fractures-dislocations with long-segment posterior pedicle fixation, which achieved solid stability with no failures of the internal fixation.

In conclusion, the current study has demonstrated that the thoracic and lumbar spine fracturedislocations were treated successfully using a single-stage procedure with posterolateral decompression, distractive reduction, and reconstructive stabilization by restoring fractured vertebral body morphology using a distractive reduction and ligamentotaxis technique, or implanting an autograft-filled titanium mesh if indicated, and long-segment posterior transpedicular constructs via a posterior-only approach. The absence of the risks related to the anterior approach, fewer complications in our series, sufficient 
circumferential decompression, maintenance of deformity correction, and stable mechanical reconstruction observed at the latest follow-up evaluation support this single-stage posterior-only approach strategy.

\section{Abbreviations}

TLICS

Thoracolumbar Injury Classification and Severity Score; ASIA

American Spinal Injury Association;

VAS

Visual Analog Scale;

ODI

Oswestry Disability Index score;

CSF

Cerebrospinal Fluid;

PLIF

Posterior Lumbar Interbody Fusion.

\section{Declarations}

\section{Ethical Approval and consent to participate}

This article does not contain any studies with animals performed by any of the authors. The human participants were approved by the ethical review board of Ethics commission Tianjin Hospital. Written informed consent was obtained from all patients.

\section{Consent for publication}

All authors declared that they agree with the publication of this article.

\section{Availability of data and materials}

The datasets used and/or analyzed during this study are available from the corresponding author on reasonable request.

\section{Competing interests}

All authors declared that they have no competing interest.

\section{Funding}

This work was partly supported by grants from TianJin Youth Medicine Talents Plan. 


\section{Authors' contributions}

Experiments Designed: Changbao Chen and Xinlong Ma. Experiments Performed: Changbao Chen, and Xiaolin Zhang. Data Analysis: Changbao Chen, Xiaolin Zhang and Xinlong Ma. Manuscript Preparation: Changbao Chen and Xinlong Ma. All the authors have reviewed and approved the final manuscript.

\section{Acknowledgements}

Not applicable

\section{References}

[1] Shapiro S, Abel T and Rodgers RB. Traumatic thoracic spinal fracture dislocation with minimal or no cord injury. Report of four cases and review of the literature. J Neurosurg 2002; 96: 333-7.

[2] Vaccaro AR, Lehman RA Jr., Hurlbert, RJ, et al. A new classification of thoracolumbar injuries: the importance of injury morphology, the integrity of the posterior ligamentous complex, and neurologic status. Spine 2005; 30:2325-33.

[3] Vaccaro AR, Oner C, Kepler CK, et al. AOSpine Thoracolumbar Spine Injury Classification System: Fracture Description, Neurological Status, and Key Modifiers. Spine 2013; 38:2028-37.

[4] Vialle R, Rillardon L, and Feydy A. Spinal trauma with a complete anterior vertebral body dislocation: A report of three cases. Spinal Cord 2008; 46:154-8.

[5] Wood KB, Li W, Lebl DR, et al. Management of thoracolumbar spine fractures. Spine J 2014; 14:14564.

[6] Lenke LG, Sides BA, and Koester LA. Vertebral column resection for the treatment of severe spinal deformity. Clin Orthop Relat Res 2010; 468:687-99.

[7] Tomita K, Kawahara N, and Murakami H. Total en bloc spondylectomy for spinal tumors: Improvement of the technique and its associated basic background. J Orthop Sci 2006; 11:3-12.

[8] Haiyun Y, Rui G, Shucai D, et al. Three-column reconstruction through single posterior approach for the treatment of unstable thoracolumbar fracture. Spine (Phila Pa 1976) 2010; 35:E295-302.

[9] Sasani M, Ozer AF. Single-stage posterior corpectomy and expandable cage placement for treatment of thoracic or lumbar burst fractures. Spine (Phila Pa 1976) 2009; 34:E33-40.

[10] Burns S, Biering-Sørensen F, Donovan W, et al. International standards for neurological classification of spinal cord injury, revised 2011. Top Spinal Cord Inj Rehabil 2012;18:85-99.

[11] Keynan O, Fisher CG, Vaccaro A, et al. Radiographic measurement parameters in thoracolumbar fractures: a systematic review and consensus statement of the spine trauma study group. Spine 2006, 
[12] Walsh TL, Hanscom B, Lurie JD, et al. Is a condition-specific instrument for patients with low back pain/leg symptoms really necessary? The responsiveness of the Oswestry Disability Index, MODEMS, and the SF-36. Spine 2003, 28:607-15.

[13] Vaccaro AR, Lim MR, Hurlbert RJ, et al. Surgical decision making for unstable thoracolumbar spine injuries: results of a consensus panel review by the Spine Trauma Study Group. J Spinal Disord Tech 2006, 19:1-10.

[14] Jazini E, Carreon LY, Dimar li JR, et al. Management of Unstable Fracture-Dislocations of the Spine in Polytrauma Patients. Instructional course lectures 2018; 67:369-76.

[15] McDonough PW, Davis R, Tribus C, et al. The management of acute thoracolumbar burst fractures with anterior corpectomy and Z-plate fixation. Spine 2004; 29:1901-8.

[16] P Oprel P, Tuinebreijer WE, Patka P, et al. Combined anterior-posterior surgery versus posterior surgery for thoracolumbar burst fractures: A systematic review of the literature. Open Orthopaedics Journal 2010; 4:93-100.

[17] Suk SI, Kim JH, Lee SM, et al. Anterior-posterior surgery versus posterior closing wedge osteotomy in posttraumatic kyphosis with neurologic compromised osteoporotic fracture. Spine (Phila Pa 1976) 2003; 28:2170-5.

[18] Lubelski D, Abdullah KG, Steinmetz MP, et al. Lateral extracavitary, costotransversectomy, and transthoracic thoracotomy approaches to the thoracic spine: review of techniques and complications. $J$ Spinal Disord Tech 2013; 26:222-32.

[19] Yadla S, Lebude B, Tender GC, et al. Traumatic spondyloptosis of the thoracolumbar spine. J Neurosurg Spine 2008; 9:145-51.

[20] Lu DC, Lau D, and Lee JG. The transpedicular approach compared with the anterior approach: An analysis of 80 thoracolumbar corpectomies. J Neurosurg Spine 2010; 12:583-91.

[21] Schmid R, Krappinger D, Seykora P, et al. PLIF in thoracolumbar trauma: Technique and radiological results. Eur Spine J 2010; 19:1079-86.

[22] Lazaro BC, Deniz FE, Brasiliense LB, et al. Biomechanics of thoracic short versus long fixation after 3column injury. J Neurosurg Spine 2011; 14:226-34.

[23] McLain RF. The biomechanics of long versus short fixation for thoracolumbar spine fractures. Spine (Phila Pa 1976) 2006; 31: S70-79.

\section{Tables}


Due to technical limitations, all tables are only available for download from the Supplementary Files section.

\section{Figures}

Fig. 1

A

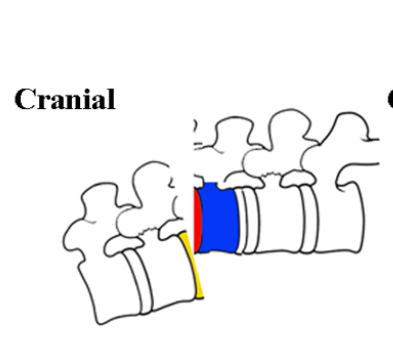

E

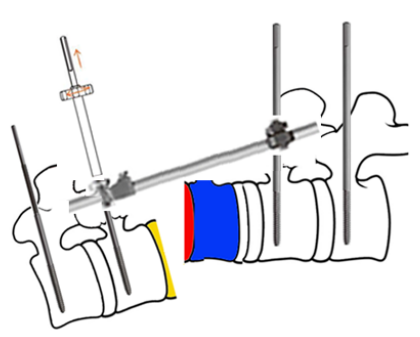

B

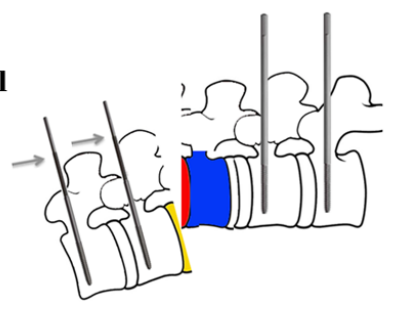

$\mathbf{F}$

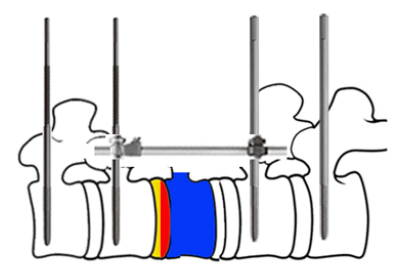

I

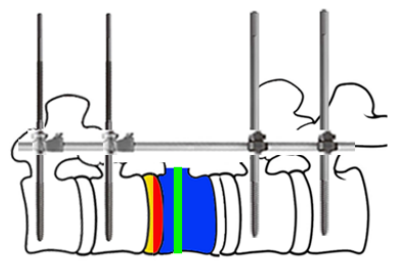

C

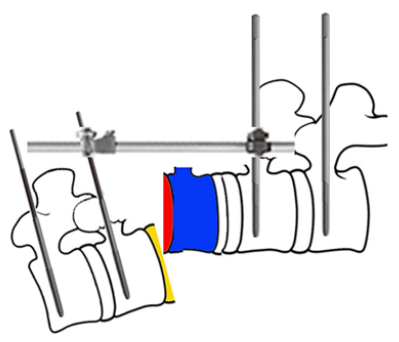

G

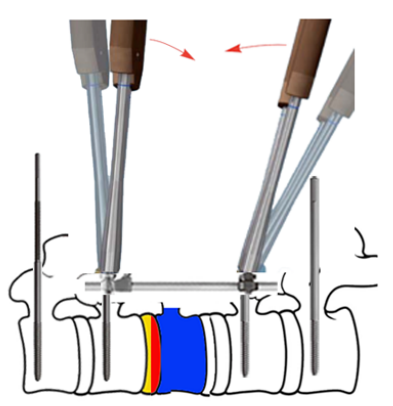

$\mathbf{J}$

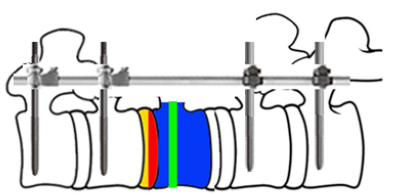

D

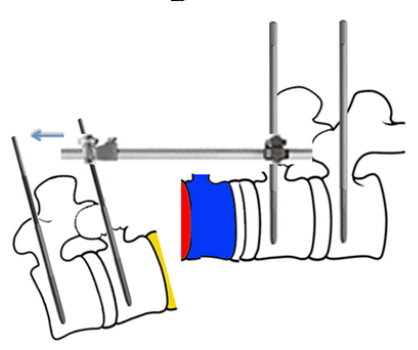

$\mathbf{H}$

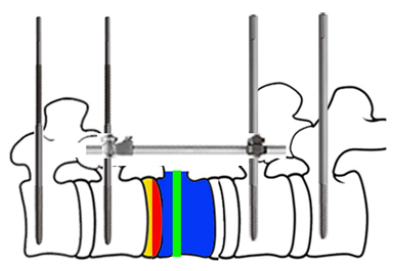

\section{Figure 1}

Schematic illustration of posterolateral decompression, distractive reduction and reconstructive stabilization for the management of the thoracic and lumbar spine fracture-dislocations through the disc. A. A schematic diagram of the thoracic and lumbar spine fracture-dislocations mainly through the disc. The cranial (colored yellow) and caudal parts (colored red) of the disrupted disc, generally coupled with the caudal vertebral body fracture (colored blue) were shown. B. Pedicle screws implanted: after the vertebral posterior arches and facet joints were fully exposed, the transpedicular Schanz screws were bilaterally implanted. Note the cranial transpedicular Schanz screws with Double Thread (arrow). C. Temporary rod assembled: after the posterior arch and facet joint were resected and partial laminectomies of the upper and lower dislocated vertebra were performed, the short temporary rod was assembled on the both sides. D. Distraction to identify the desired gap to facilitate reduction of dislocated 
vertebra. E. Reduction of the dislocated vertebra: after posterolateral decompression and release, a partial reduction of the sagittal deformity was already noticed, secure the caudal and cranial fracture clamps to the temporary rod, and the desired reduction is achieved. F. Complete reduction of the dislocated vertebra. G. Place the cannulated socket wrenches bilaterally over the cranial and caudal Schanz screws and clamps. Tilt both wrenches cranially and caudally to reduce the anterior column and lordose the spine, respectively. Secure the desired angulation by tightening the nut on the clamp with the socket wrench. $\mathrm{H}$. Restoration of the fractured vertebral body height and correction of kyphotic deformity. Note the colored green part of the vertebral body fracture represents the restored vertebral body height. I. The final titanium rods with appropriate length were installed on both sides. J. Fix fracture clamps on the final rods and trim Schanz screws.

Fig. 2

A

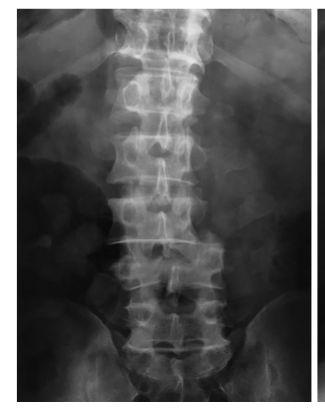

B

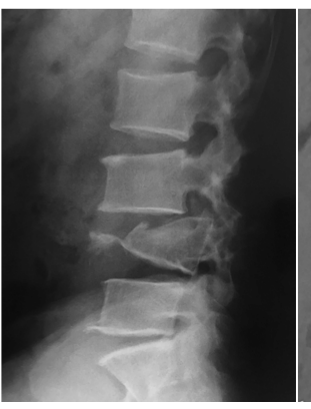

C

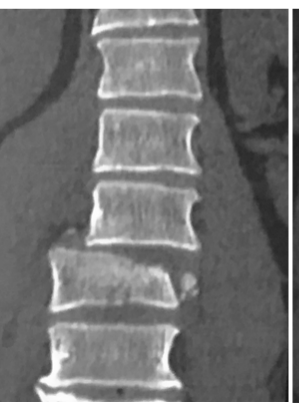

D

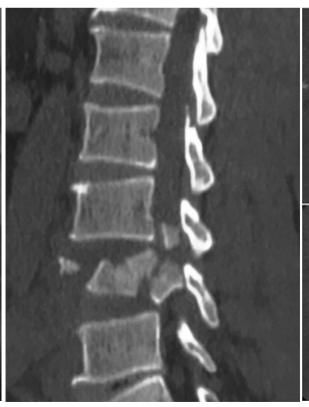

E

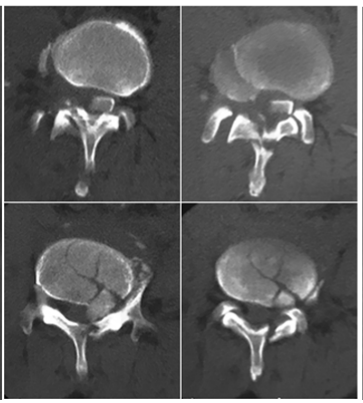

F

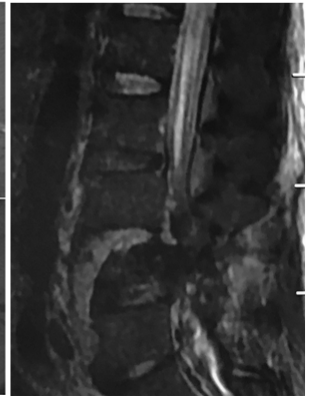

G

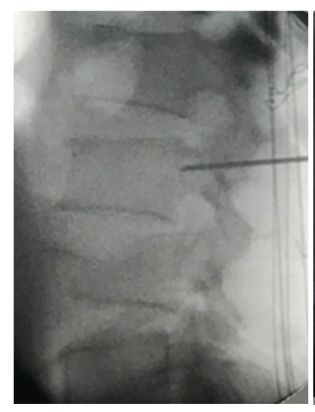

L

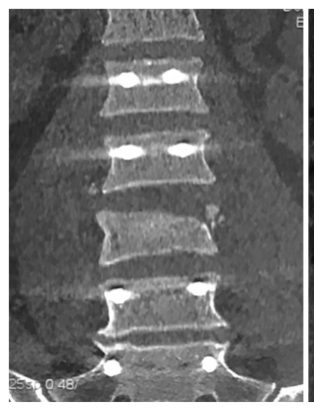

H

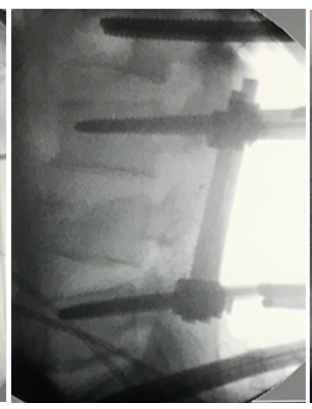

M

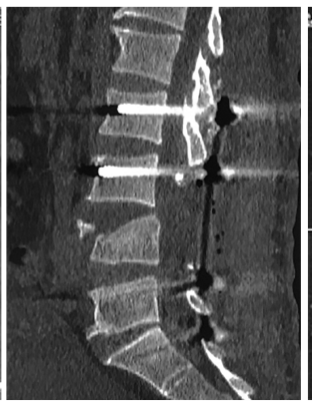

I

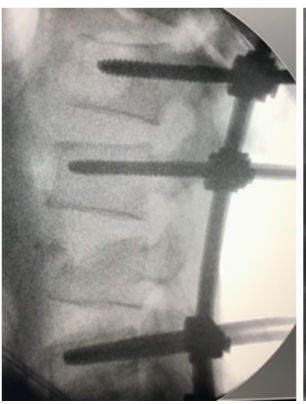

$\mathbf{N}$

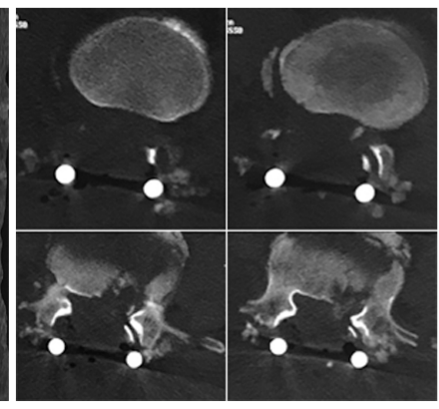

J

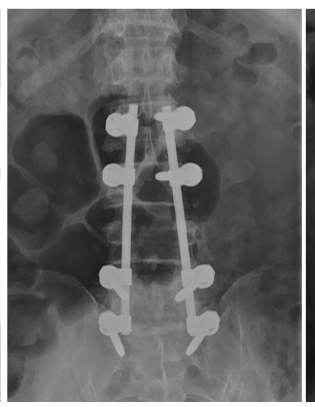

o

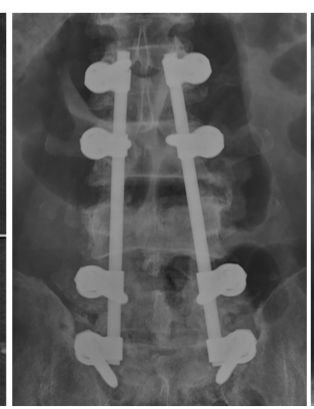

K

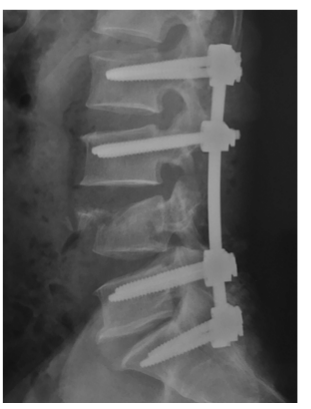

P

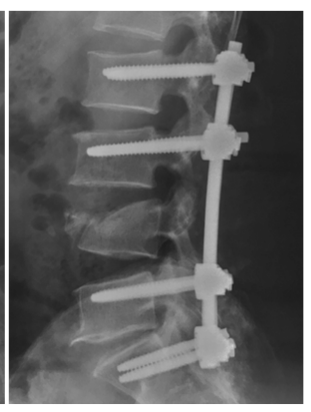

Figure 2

A 56-year-old man (Case 4) sustained L3/4 fracture-dislocations in a traffic accident, and presented with L3/4 disc disruption and L4 burst fracture causing dislocated dislocation. Posterolateral decompression and distractive reduction through a posterior-only approach were successfully accomplished at 2 days 
after trauma. Preoperative imaging of frontal and lateral X-ray (A, B), CT scans (C, D, E), and MRI (F) showing the lumbar spine fracture-dislocations through L3/4 disc and anterior telescoping. Intraoperative imaging of fluoroscopy illustrating Kirschner wire positioning and L3 anterior dislocation relative to L4 vertebral body $(G)$, complete reduction of anterior dislocated L3 vertebra using the short temporary rod after posterolateral decompression and release $(\mathrm{H})$, the final titanium final rods with appropriate length were precontoured to the appropriate anatomic sagittal profile and were installed on both sides (I). Postoperative imaging of frontal and lateral X-ray $(\mathrm{J}, \mathrm{K})$, coronal $(\mathrm{L})$, sagittal $(\mathrm{M})$ reconstruction, and axial $\mathrm{CT}$ scan $(\mathrm{N})$ demonstrating a full decompression, a complete reduction, and coronal and sagittal alignment restored. The latest follow-up imaging of frontal and lateral X-ray $(\mathrm{O}, \mathrm{P})$ showing a complete reduction, a fine anterior column reconstruction and an excellent coronal and sagittal alignment.

\section{Fig. 3}

A

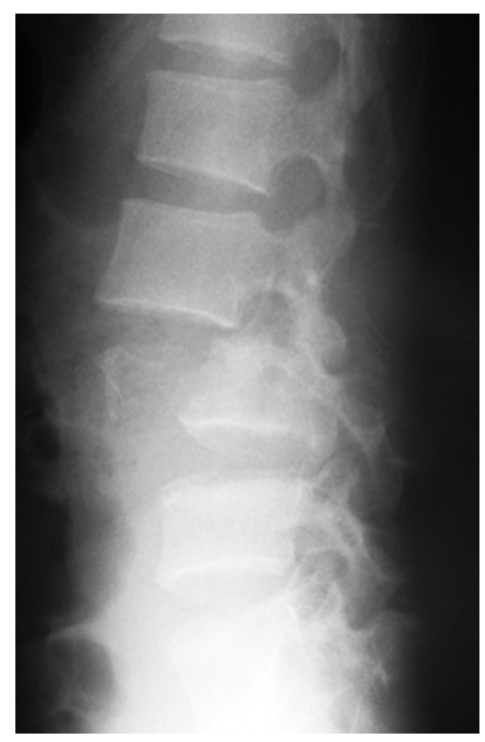

E

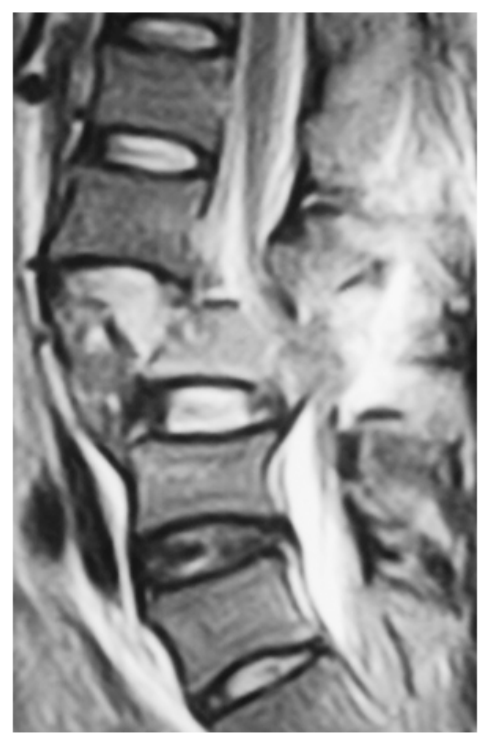

B

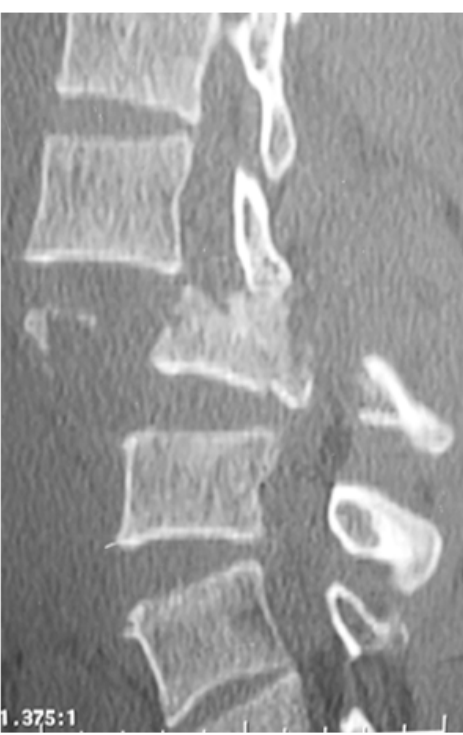

F

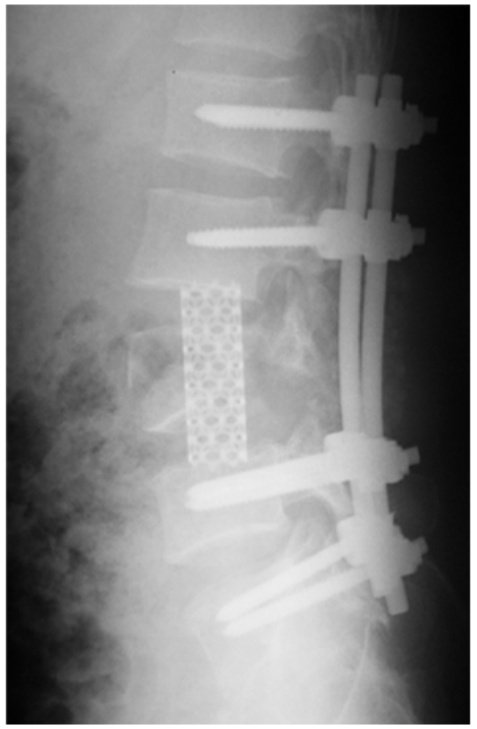

C

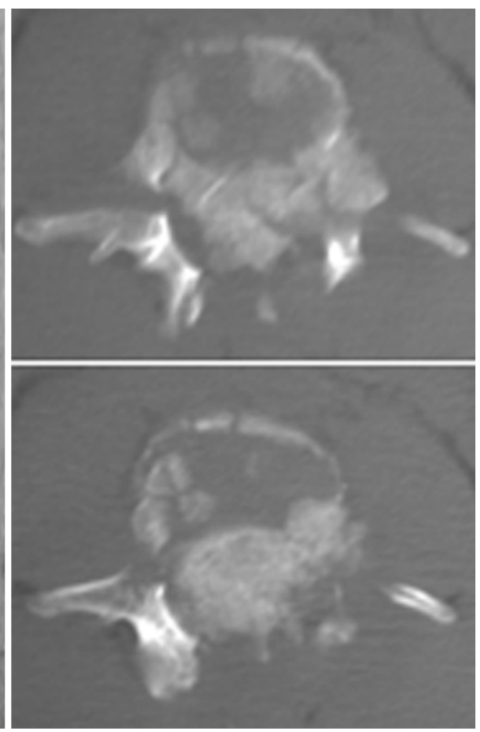

G

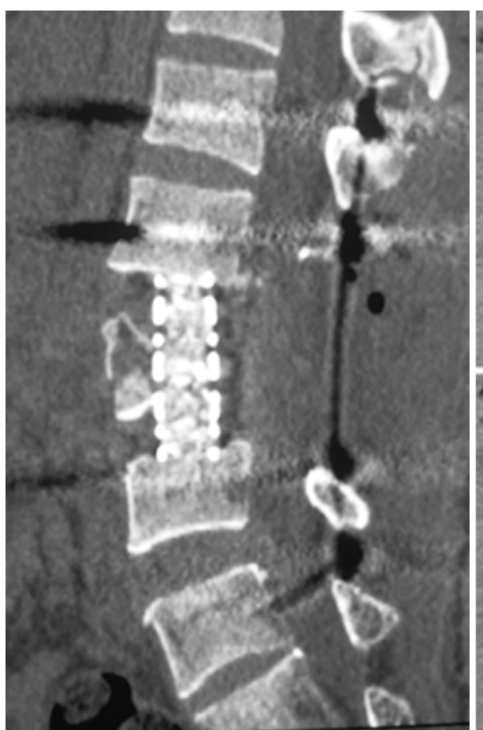

D

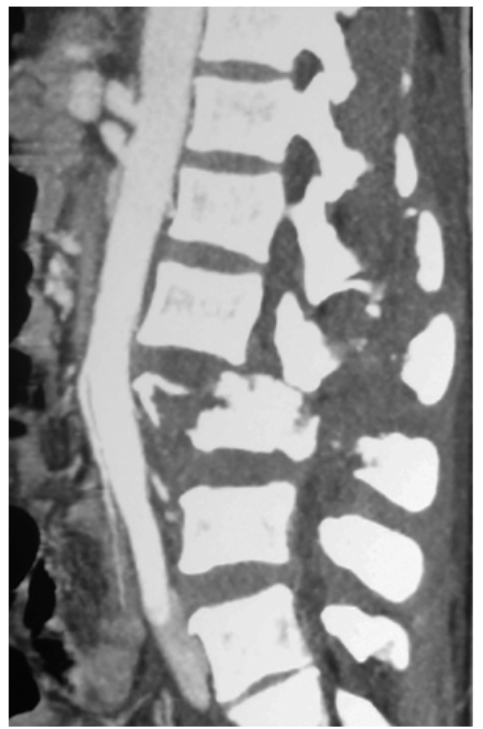

H

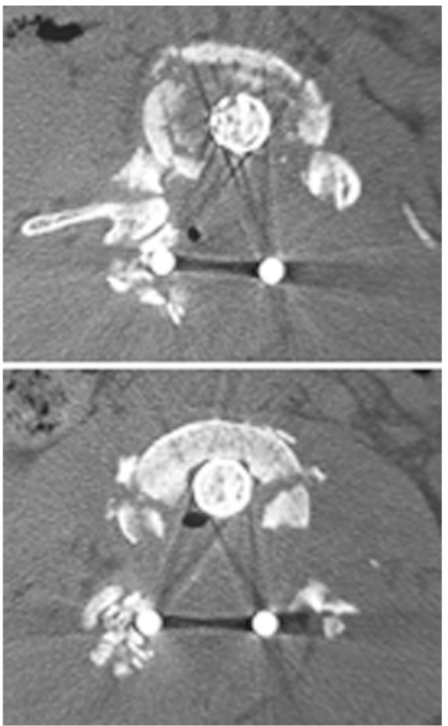

Figure 3 
A 35-year-old man (Case 19) presented with L2/3 disc disruption and L3 severe burst fracture with significantly comminuted and conspicuous fragment displacement causing anterior dislocation of L2 vertebra relative to L3 vertebral body after heavy blow. Posterolateral decompression and L3 corpectomy, and distractive reduction through a posterior-only approach were successfully accomplished at 22 days after trauma. Preoperative imaging of X-ray (A), CT scans (B, C), CT angiography (D) and MRI (E) showing dislocated dislocation of the $L 2$ vertebra and $L 3$ severe burst fracture through $L 2 / 3$ disc and anterior telescoping. Postoperative imaging of lateral X-ray $(F)$, sagittal reconstruction $(H)$ and axial $(\mathrm{I}) \mathrm{CT}$ scan demonstrating a full decompression, L3 corpectomy and anterior column reconstruction, and a perfect sagittal alignment.

\section{Supplementary Files}

This is a list of supplementary files associated with this preprint. Click to download.

- Tables20191018.docx 